\title{
THE AMALGAMATION PROPERTY FOR $G$-METRIC SPACES
}

\author{
H. H. HUNG
}

\begin{abstract}
Let $G$ be a (totally) ordered (abelian) group. A $G$ metric space $(X, \rho)$ consists of a nonempty set $X$ and a $G$-metric $\rho$ : $X \times X \rightarrow G$ (satisfying the usual axioms of a metric, with $G$ replacing the ordered group of real numbers). That the amalgamation property holds for the class of all metric spaces is attributed, by Morley and Vaught, to Sierpiński. The following theorem is proved. THEOREM. The class of all G-metric spaces has the amalgamation property if, and only if, $G$ is either the ordered group of the integers or the ordered group of the reals.
\end{abstract}

The purpose of this paper is to prove that the amalgamation property fails for the class of all $G$-metric spaces, for every (totally) ordered (abelian) group $G$ which is not equal to the additive group of the integers or that of the real numbers.

The amalgamation property, in its abstract form, was first formulated by Fraïssé [3] in connection with embedding problems. It has been studied by Jónsson [6], [7], [8] and [9], and Morley and Vaught [12] in connection with the general theory of homogeneous-universal structures in Jónsson classes.

Not too many examples of classes of relational systems are known for which the amalgamation property fails. Among them the following are included: (a) the class of semigroups and hence of rings (Kimura [11], Jónsson [6], [7], Howie [5]); (b) the class of modular lattices (Jónsson [10]); (c) the class of l-groups (Pierce [13]). To these, because of our theorem, we can now add a whole family of such examples, among which is the class of all metric spaces on which the metric takes on only rational values.

Classes of metric spaces were studied by Urysohn [17], [18] and Sierpiński [14], [15] from the point of view of the existence of universal

Presented to the Society, July 6, 1971; received by the editors April 25, 1972.

AMS (MOS) subject classifications (1970). Primary 06A55, 08A05, 54E35.

Key words and phrases. Amalgamation property, relational systems, totally ordered abelian groups, order completeness, Dedekind cuts, $G$-metric spaces.

${ }^{1}$ The author is partially supported by NRC Grant A4035. This work is a part of a Ph.D. Thesis written at McGill University under the direction of Professor S. Negrepontis, to whom the author is deeply grateful.

(C) American Mathematical Society 1973 
spaces; thus, Urysohn proved the existence of a universal separable, complete and $\omega$-homogeneous metric space, unique up to isometries; while Sierpinski proved that for each cardinal $\alpha$ such that $2^{\omega} \leqq \alpha=2^{\alpha}$ $\left(=\sum\left\{2^{\beta}: \beta<\alpha\right\}\right)$, there exists a universal metric space of cardinality $\alpha$. It was implicitly proved by Sierpiński in [15], as pointed out by Morley and Vaught [12], that the class of all metric spaces has the amalgamation property (and in fact forms a Jónsson class).

The notion of a metric has been generalized by replacing the additive group of real numbers by an arbitrary (totally) ordered (abelian) group $G$ (cf. e.g. Hausdorff [4], Cohen and Goffman [1], [2] and Sikorski [16]), and it is easy to see that the result of Sierpiński, and Morley-Vaught on the amalgamation property extends to $G$-metric spaces whenever $G$ is order complete. The main result of this paper is the converse statement.

This paper is organized as follows: In $\S 1$ we establish the necessary notations and mention standard definitions and results; in $\$ 2$ three simple lemmas are established; in $\S 3$ the proof of the main theorem is given.

1. Preliminaries. A (totally) ordered (abelian) group $G$ is an additive group on which is defined an order relation $<$ such that if $a<b$, then $a+c<b+c$, for all $a, b, c \in G$. We write $\leqq$ for $<$ or $=$, and denote by $G^{+}$the set of nonnegative elements of $G$. Two positive elements $x, y$ of an ordered group are relatively archimedean if there are positive integers $m$, $n$ such that $m x \geqq y$ and $n y \geqq x$. If every two positive elements of an ordered group are relatively archimedean, then the ordered group is archimedean. Every archimedean ordered group is isomorphic to an ordered subgroup of the additive group of the real numbers. An ordered group $G$ is order complete if every nonvoid subset of $G$ that has an upper bound has a least upper bound. A Dedekind cut $X \mid Y$ in ordered group $G$ is a pair of nonvoid subsets $X, Y$ of $G$ such that (i) $X \cup Y=G$, (ii) $x<y$ for all $x \notin X$ and $y \in Y$, and (iii) $X$ has no last and $Y$ has no first element. A Dedekind cut $X \mid Y$ is positive if $0 \in X$. Every ordered group that is not order complete has a positive Dedekind cut.

Given an ordered group $G$, a $G$-metric on a nonempty set $X$ is a symmetric nonnegative function $\rho$ from $X \times X$ into $G$ such that $\rho(x, y)=0$ if and only if $x=y$ and such that the triangle inequality is satisfied; the pair $(X, \rho)$ is a $G$-metric space. (If $G=\boldsymbol{R}$ (the ordered group of real numbers), then the notion is reduced to that of a metric space.) A $G$-isometry has the obvious meaning.

For a given group $G$, the class $\mathscr{K}_{G}$ of $G$-metric spaces is said to have the amalgamation property if given $A, B, C \in \mathscr{K}_{G}$ and given $G$-isometries $f, g$ of $C$ into $A, B$ respectively, there are $D \in \mathscr{K}_{G}$ and $G$-isometries $f^{\prime}, g^{\prime}$ of $A, B$ respectively $D$ into such that $f^{\prime} \circ f=g^{\prime} \circ g$. 
From each $G$-metric space $(X, \rho)$ we can obtain a relational system $\left\langle X, R_{g}\right\rangle_{g \in G^{+}}$where $R_{g} x y$ if and only if $\rho(x, y)=g$ (cf. [12]). That $\mathscr{K}_{G}$ has the amalgamation property is equivalent to the class of relational systems thus obtained having the amalgamation property in the usual sense.

2. Some lemmas. A positive Dedekind cut $X \mid Y$ will be called archimedean if there exist $x_{1}, x_{2} \in X$ such that $x_{1}+x_{2} \in Y$. We shall write, for all subsets $A$ of an ordered group $G,[A]$ for $\{x \in G: a<x$ for some $a \in A\}$ and $2 A$ for $\{2 a: a \in A\}$. A positive Dedekind cut $X \mid Y$ will be called quotient if $G \sim[2 Y] \mid[2 Y]$ is also a Dedekind cut. To show that $X \mid Y$ is quotient it suffices to show that $G \sim[2 Y]$ has no last element. The next three simple lemmas will lead up to the main theorem (proved in §3).

2.1. LEMMA. Every archimedean ordered group that is not order complete has a quotient positive Dedekind cut.

This lemma is evident if we make the following observations: Every archimedean ordered group $G$ that is not order complete can be assumed to be a dense subgroup of the additive group of the reals; the Dedekind cuts are in (1-1) correspondence with the elements of $R \sim G$; the cut corresponding to an $x \in R \sim G$ is positive and quotient if and only if $x$ is positive and $2 x$ is not a member of $G$.

2.2. LEMMA. Every nonarchimedean ordered group has a nonarchimedean positive Dedekind cut.

Proof. Let $G$ be a nonarchimedean ordered group. Let $x, y, 0<x<y$, be relatively nonarchimedean. Let

$$
\begin{aligned}
X & =\{z \in G: n z<y, \text { for all natural numbers } n\}, \\
Y & =\{z \in G: n z \geqq y, \text { for some natural number } n\} .
\end{aligned}
$$

Clearly $X$ and $Y$ are nonempty such that (i) $X \cup Y=G$, (ii) $a<b$ for all $a \in X, b \in Y$, and $0 \in X$. Further, $X$ has no last element. For, if $x_{0} \in X$ is the last element, then since $2 x_{0}>x_{0}>0$, it follows that $2 x_{0} \in Y$ and $x_{0} \notin X$ by the definition of $X$. Also, $Y$ has no first element. For, if $y_{0} \in Y$ is the first element, $y_{0}-x$, being smaller than $y_{0}$, is in $X$. But $\left(y_{0}-x\right)+x \in Y$ which implies either $y_{0}-x$ or $x$ is in $Y$ contrary to our assumption. Therefore $X \mid Y$ is a positive Dedekind cut. Its being nonarchimedean is evident from its definition.

2.3. LEMMA. Everynonarchimedean ordered group has a quotient positive Dedekind cut.

Proof. Let $G$ be a nonarchimedean ordered group. Let $X \mid Y$ be a nonarchimedean positive Dedekind cut. We shall prove it to be quotient by 
showing that $G \sim[2 Y]$ has no last element. Suppose $G \sim[2 Y]$ has a last element $z$. Let $x$ be a positive element in $X$. Then $z+2 x>z$ implies that $z+2 x$ is not in $G \sim[2 Y]$. Thus, $z+2 x \geqq 2 y$ for some $y$ in $Y$. Hence, $z \geqq 2(y-x)$. This is a contradiction, because $X \mid Y$ being nonarchimedean implies that $y-x$ belongs to $Y$.

3. Main result. We are now ready to state and prove the main result of this paper.

THEOREM. Let $G$ be a (totally) ordered (abelian) group. The class $\mathscr{K}_{G}$ of all G-metric spaces satisfies the amalgamation property, if and only if $G$ is either the ordered group of the integers or that of the real numbers.

Proof. As is well known (and easy to prove), an ordered group $G$ is order complete if and only if $G$ is either the ordered group of the integers, $\boldsymbol{Z}$ or that of the real numbers, $\boldsymbol{R}$. As it was mentioned in the introduction, it is known that the amalgamation property holds for $\mathscr{K}_{R}$; the proof, which uses the order completeness of $\boldsymbol{R}$, carries over to every order complete group. We outline a proof of the statement that $\mathscr{K}_{G}$ satisfies the amalgamation property for every order complete group $G$.

Let $A, B$ and $C$ be any $G$-metric spaces with $G$-metrics $\alpha, \beta$ and $\gamma$ respectively. Let there be $G$-isometries $f: C \rightarrow A, g: C \rightarrow B$. Let $E$ be the disjoint union of the sets $A$ and $B$. We define $\varepsilon: E \times E \rightarrow G$ as follows. For $a, b \in E$,

$$
\begin{aligned}
\varepsilon(a, b) & =\alpha(a, b) & & \text { if } a, b \in A, \\
& =\beta(a, b) & & \text { if } a, b \in B, \\
& =\inf _{c \in C}[\alpha(a, f(c))+\beta(b, g(c))] & & \text { if } a \in A, b \in B .
\end{aligned}
$$

It can be easily verified that $\varepsilon$ is $G$-pseudometric (i.e. a $G$-metric minus the requirement that $\varepsilon(x, y)>0$ if $x \neq y)$ by checking the triangle inequality.

If $(E, \varepsilon)$ is a $G$-pseudometric space, we can get a $G$-metric space $(D, \delta)$ out of it in the usual way (by identifying points of zero $\varepsilon$-distance). The $G$ metric space $(D, \delta)$, together with the $G$-isometries resulting from the natural embeddings of $A, B$ into $E$ satisfies the amalgamation property.

For the converse, let $G$ be an ordered group that is not order complete. Let $X \mid Y$ be a quotient positive Dedekind cut which exists according to Lemmas 2.1 and 2.3. We are to construct three $G$-metric spaces $(A, \alpha)$, $(B, \beta)$ and $(C, \gamma)$ together with $G$-isometries $f: C \rightarrow A, g: C \rightarrow B$ such that there does not exist a $G$-metric space $(D, \delta)$ with $G$-isometries $f^{\prime}, g^{\prime}$ from $A$ and $B$ respectively into it such that $f^{\prime} \circ f=g^{\prime} \circ g$.

Choose an arbitrary $y_{0} \in Y$. Let $Z=\left\{-2 y_{0}-z: z \in G \sim[2 Y], z \geqq 0\right\}$. Let $C=Y \dot{\cup} Z$ (the disjoint union of $Y$ and $Z$ ), and define the $G$-metric $\gamma$ on $C$ 
as follows:

$$
\begin{aligned}
\gamma(a, b) & =|a-b|, \quad \text { if } a, b \in Y \text { or } a, b \in Z, \\
& =2 y_{0}+a, \quad \text { if } a \in Y, b \in Z .
\end{aligned}
$$

Let $A=\left\{0_{A}\right\} \dot{\cup} C$, and define the $G$-metric $\alpha$ on $A$ as follows:

$$
\begin{aligned}
\alpha(a, b) & =\gamma(a, b), & & \text { if } a, b \in C, \\
& =b, & & \text { if } a=0_{A}, b \in Y, \\
& =2 y_{0}, & & \text { if } a=0_{A}, b \in Z .
\end{aligned}
$$

Let $B=\left\{0_{B}\right\} \cup \dot{\cup}$, and define the $G$-metric $\beta$ on $B$ as follows:

$$
\begin{aligned}
\beta(a, b) & =\gamma(a, b), & & \text { if } a, b \in C \\
& =b, & & \text { if } a=0_{B}, b \in Y \\
& =-b, & & \text { if } a=0_{B}, b \in Z .
\end{aligned}
$$

Let $f, g$ be the natural embeddings of $C$ into $A, B$ respectively.

Readers can verify that $A, B, C$ are indeed $G$-metric spaces by checking the triangle inequality in detail.

The natural embeddings $f, g$ are $G$-isometries by definition.

Now we show that there does not exist a $G$-metric space $(D, \delta)$ with $G$-isometries $f^{\prime}$ and $g^{\prime}$ from $A$ and $B$ respectively into it such that $f^{\prime} \circ f=$ $g^{\prime} \circ g$.

Suppose the contrary. Let $\delta\left(f^{\prime}\left(0_{A}\right), g^{\prime}\left(0_{B}\right)\right)=\Delta$. We can prove $\Delta \in G \sim$ [2Y]. For, $\Delta \leqq \delta\left(f^{\prime}\left(0_{A}\right), f^{\prime}(y)\right)+\delta\left(g^{\prime}\left(0_{B}\right), g^{\prime}(y)\right)=2 y$, for all $y \in Y$, and therefore by definition of $[2 Y], \Delta \in G \sim[2 Y]$. There then exists $z_{0} \in G \sim$ $[2 Y]$ such that $z_{0}>\Delta$.

If we consider the triangle $f^{\prime}\left(0_{A}\right) g^{\prime}\left(0_{B}\right) f^{\prime}\left(-2 y_{0}-z_{0}\right)$ on $D$, we will find a contradiction to the triangle inequality as follows:

$$
\begin{aligned}
\delta\left(f^{\prime}\left(0_{A}\right), f^{\prime}\left(-2 y_{0}-z_{0}\right)\right) & =2 y_{0}, \\
\delta\left(g^{\prime}\left(0_{B}\right), f^{\prime}\left(-2 y_{0}-z_{0}\right)\right) & =2 y_{0}+z_{0}, \\
\delta\left(f^{\prime}\left(0_{A}\right), g^{\prime}\left(0_{B}\right)\right) & =\Delta,
\end{aligned}
$$

and $2 y_{0}+\Delta 2 y_{0}+z_{0}$. This completes the proof of the theorem.

\section{REFERENCES}

1. L. W. Cohen and C. Goffman, The topology of ordered Abelian groups, Trans. Amer. Math. Soc. 67 (1949), 310-319. MR 11, 324.

2. - On the metrization of uniform space, Proc. Amer. Math. Soc. 1 (1950), 750-753. MR 12, 434.

3. R. Fraïssé, Sur l'extension aux relations de quelques propiriétés des ordres, Ann. Sci. Ecole Norm. Sup. (3) 71 (1954), 363-388. MR 16, 1006. 
4. F. Hausdorff, Grundzüge der Mengenlehre, Veit, Leipzig, 1914; photographic reproduction, Chelsea, New York, 1949. MR 11, 88.

5. J. M. Howie, Embedding theorems with amalgamation for semigroups, Proc. London Math. Soc. (3) 12 (1962), 511-534. MR 25 \#2139.

6. B. Jónsson, Universal relational systems, Math. Scand. 4 (1956), 193-208. MR 20 \#3091.

7. - Homogeneous universal relational systems, Math. Scand. 8 (1960), 137-142. MR 23 \#A2328.

8. - Algebraic extensions of relational systems, Math. Scand. 11 (1962), 179-205. MR $27 \# 4777$.

9. - Extensions of relational structures, Theory of Models (Proc. 1963 Internat. Sympos. Berkeley), North-Holland, Amsterdam, 1965, pp. 146-157. MR 34 \#2463.

10. The amalgamation property in varieties of modular lattices, Notices Amer. Math. Soc. 18 (1971), 400. Abstract \#71T-A42.

11. N. Kimura, On semigroups, Doctoral Dissertation, Tulane University, New Orleans, La., 1957.

12. M. Morley and R. Vaught, Homogeneous universal models, Math. Scand. 11 (1962), 37-57. MR 27 \#37.

13. K. R. Pierce, Amalgamations of lattice ordered groups, Notices Amer. Math. Soc. 18 (1971), 946. Abstract \#71T-A240.

14. W. Sierpiński, Sur un espace métrique séparable universel, Fund. Math. 33 (1945), 115-122. MR 7, 420.

15. - Sur les espaces métriques universels, Fund. Math. 33 (1945), 123-136. MR 7, 420.

16. R. Sikorski, Remarks on some topological spaces of high power, Fund. Math. 37 (1950), 125-136. MR 12, 727.

17. P. Urysohn, Sur un espace métrique universal, C. R. Acad. Sci. Paris 180 (1925), 803-806.

18. —_ Sur un espace métrique universel, Bull. Sci. Math. (2) 51 (1927), 43-64.

Department of Mathematics, McGill University, Montréal, Québec, Canada

Current address: Department of Mathematics, Sir George Williams University, Montréal, Québec, Canada 